\title{
Adaptation in the constancy of visual direction tested by measuring the constancy of auditory direction'
}

\author{
HANS WALLACH AND JEROME H. KRAVITZ, 2 \\ SWARTHMORE COLLEGE
}

\begin{abstract}
Modification of the constancy of visual direction was produced by partially adapting Ss to the displacements of the visual field caused by magnifying lenses during $l \mathrm{~h}$ of continuous head turning. The adaptation effects were measured by determining the range of perceived target immobility before and after this adaptation period. $A$ method for measuring the range of apparent immobility of an auditory signal during head movements was developed and employed to test whether a modification of the constancy of visual direction transfers to the constancy of auditory direction. $N_{0}$ such transfer was found, and it was concluded that a modification of the constancy of visual direction does not consist in an altered evaluation of kinesthetic cues for head turning. The method and the equipment used in the investigation of the constancy of visual direction are described; knowledge of the previous brief publications on this topic is not needed.
\end{abstract}

In previous work on the subject of this article (Wallach \& Kravitz, 1965a) we developed a method for the measurement of the constancy of visual direction. This term refers to the fact that the visual environment appears to be stationary when one turns his head. Such head movements bring about displacements between the environment and the head: turning the head to the right causes the environment to become displaced relative to the head to the left. As a condition of stimulation this displacement, taken by itself, is ambiguous; it can also be produced by an objective displacement of the environment to the left, and such an event normally causes veridical perceived motion of the environment. It is the presence or absence of the head movement during the displacement that determines whether the latter leads to perceived motion of the environment or to immobility. Our previous work shows that it is not the mere presence or absence of head turning that is here decisive; rather the amount of the head movement is, as it were, compared with the amount of displacement of the environment relative to the head. Only if that relative displacement closely approximates that which is normally produced by the head movement is the environment perceived as stationary. If it exceeds or is less than this normal amount, that is, if the environment is being objectively displaced during the head movement in the plane of the head's rotation, the environment is seen to move. In other words, the amount of head turning is normally taken into account in the perception of motion or rest of the environment; hence the use of the term constancy. Ways of measuring the accuracy with which this constancy operates are the first concern of this report.

The considerations just presented are based on the fact that stimulation that results in motion perception always consists in a relative displacement, either between several environmental objects or between an environmental object and 0 . Here we are concerned with the latter kind, a displacement of the whole environment relative to $O$. It results from objective motion of the environment about $O$, causing a displacement of the environment relative to O's head, which, in turn, produces image displacement or pursuit eye movements. Identical conditions for visual stimulation, however, will result from head rotation with the environment objectively stationary; it also will cause a displacement of the environment relative to O's head. Therefore, displacement between environment and head, by itself, is an ambiguous condition of stimulation. Were it always to cause motion perception, one would see the environment move to the left when he turns his head to the right and vice versa. The only way in which this can be prevented is by resolving the ambiguity through the use of kinesthetic information about the movement or rest of the head. Then, when visual stimulation representing the environment as being displaced is received, it will depend on kinesthetic stimulation whether this results in perceived motion of the environment or in its perceived immobility. Therefore, the stimulation necessary to see the environment at rest during a turning of the head to the right is a twofold one: a displacement relative to the head of the environment in the opposite direction, that is, to the left, and kinesthetic stimulation representing the head movement of matching amount to the right.

Our concern here is with this matching of the amount of environmental displacement and the amount of head rotation. How accurate must this match be for the environment to be perceived as stationary during a head movement? Translated into concrete terms this means: How exactly must the visual environment as a whole be stationary during a head movement to be perceived as stationary? Or, in other words, how much may the visual field be displaced during a head movement and still be perceived as stationary? A procedure by which this question can be answered is in a sense similar to the one by which the accuracy of the perception of straightness of a line would be measured by presenting $S$ with a series of, say, vertical lines, consisting of a straight line and a number of slightly curved lines varying in degree of curvature. $S$ being asked to select those lines that appear straight to him would not only choose the objectively straight line but also a number of the physically curved lines from among those with the smallest curvature and call them straight. Gibson (1933) has observed that a straight line is part of "continuous series with two opposed kinds of experiences at the ends," namely curvature convex-to-the-right and curvature convex-to-the-left, and "a unique mental experience" namely, straightness, in the middle. As, objectively, curvature of one kind decreases from one end of the series, changes into straightness and then curvature of the other kind increases toward the other end, a range of apparent straightness can be determined that comprises the objectively straight line and a number of the least curved lines of either kind of curvature. In our measurements, a series of environmental displacements of different rates occurring during repeated head movements is presented which corresponds to the series of curved lines. The displacement series consists of motion to the left, immobility, and motion to the right when the head movement is, say, to the right, with the displacement rate arranged like the degree of curvature: one kind of motion decreasing in rate toward immobility and then the other motion increasing in rate. When presented, such a series yields a range of apparent immobility of the environment that comprises objective immobility and a number of the smallest displacement rates of either kind, motion to the left and motion to the right. Other displacement rates located in the series on either side of the range of apparent immobility, will be perceived as motion.

Such measurements were taken with an apparatus ${ }^{3}$ that transmitted S's head rotation to a luminous target that, being presented in an otherwise dark visual field, stood for the visual environment. The amount of target motion in relation to the head rotation and the direction of the target motion in relation to the direction of the head rotation were variable and under the control of E. Speed of the head rotation and its extent were left to the individual $\mathrm{S}$, who was asked to turn his head back and forth in a natural manner. With a setting made by $E$ the apparatus would displace the target direction by a constant fraction of the head rotation. Therefore, for a given setting, the ratio of the angle through which the target was displaced to the angle through which the head was turned was constant. The absolute amount of target displacement and its speed depended partly on the speed and 


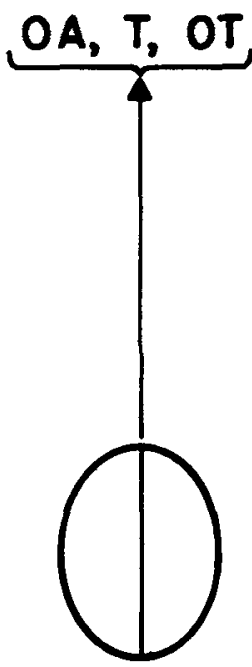

(a)

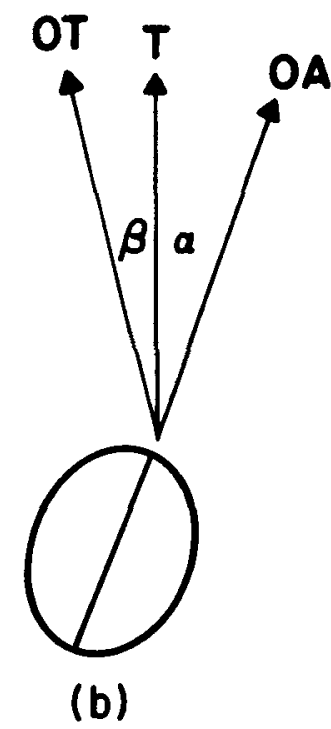

Fig. 1. Magnification causes field displacement during head movements. The enlargement of all visual angles in magnification causes a shift of all visual directions away from the optical axis $(\mathrm{OA})$ in proportion to the angle they form with $O A$ and to the power (p) of magnification. In (a) the arrow is at once the optical axis of the magnifier $(O A)$, the objective tanget direction $T$, and the optical target direction (OT). (b) After a clockwise turning of the head in the amount of $\alpha, O A$ is in a new position. $T$, which is, of course, unchanged, now forms an angle with $O A$ equal to $\alpha$. This angle is being magnified $(\alpha \cdot p)$ and this magnified angle is the one that the new optical target direction (OT) forms with OA. The difference between OT and $T(\beta)$ is the optical displacement of the target direction due to the head movement. $\beta=\alpha \cdot p-\alpha$.

extent of the head movements, but $E$, by setting the apparatus for particular ratios of target displacement to rotational head displacement, fully controlled the relative rate of target displacement. This proportion of target to head displacement was called displacement ratio (DR for short) and, transformed into per cent by multiplying by 100 , was the unit of all our measurements. This unit (\% DR) corresponds to degree of curvature. There are two ways in which the target can be displaced in relation to a head movement: with the head rotation (target moves to right when head turns right) or against it. They correspond to the two kinds of curvature on either side of the straight line, which corresponds in our case to a motionless target.

Just as the range of apparent straightness which comprises the straight line and a number of the least curved lines of both kind of curvature, measures sensitivity to objective curvature, so a range of displacement ratios that lead to perception of immobility measures the sensitivity to target displacement during head movements. Normally, such a "no-motion" range comprises objective target rest and small displacements with and against the head. We found a surprisingly narrow no-motion range; a target displacement of $4 \%$ DR to either side of objective target rest was on the average enough for target motion to be consistently reported.

Such measurements make it possible to investigate adaptation to spectacles that optically displace the visual field during a head movement. That such adaptation occurs has been known since Stratton in 1896 wore inverting lenses, which by switching optically left and right, cause a strong displacement of the visual field when the head is being turned. They change the normal displacement of the field relative to the head which is to the left when the head turns to the right into one to the right. Instead of the displacement of the field relative to the head against the head rotation, the optical displacement is with the head movement and results in a perceived swinging of the visual field when the head turns. Stratton found that such perceived swinging of the field with the head rotation eventually stops and that swinging against the head rotation is observed when the spectacles are tiken off. For reasons previously explained (Wallach \& Kravitz, 1965a), we avoided optical left-right reversal in our study of adaptation in the constancy of visual direction. Instead we produced optical displacement of the visual field during head movements by magnifying or minifying lenses.

Magnification causes field displacement during head movements in the following manner. A magnifier optically enlarges all visual angles in proportion to its power (p). When the head is turned by a certain angle, the direction of a stationary object normally becomes displaced relative to the head by the same angle $(\alpha)$. This angle, however, is being magnified $(\alpha \cdot p)$. The excess of the magnified displacement $(\alpha \cdot p)$ over the normal displacement $(\alpha)$ of a stationary object is the optical displacement $(\beta)$ of the direction of the object. Hence $\beta=\alpha \cdot p-d$, or, $\beta=\alpha(p-1)$. Thus, the field displacement during a head movement depends on the power of the magnifier and is proportional to the head rotation (see also Fig. 1). It follows that the ratio of field displacement to the rotational displacement of the head equals the magnifying power minus one. A magnifier of 1.8 power, e.g., produces a target displacement of $80 \% \mathrm{DR}$. Inasmuch as the optical displacement is caused by the magnification enlarging the normal displacement between field and head during head movements and since this normal displacement is against the head rotation, the optical displacement (which is equivalent to an objective displacement) is also against the head movements. In minification where the power is smaller than one and the normal field displacement relative to the head is diminished, the optical target displacement is with the head movements.

Our first adaptation effects were produced by having Ss wear minifying spectacles of .66 power. They caused an optical displacement of the visual field by $34 \%$ DR with the head movement, i.e., the normal field displacement relative to the turning head, which equals the head rotation, is changed into one that amounts to only $66 \%$ of the normal displacement. This condition leads to a perceived motion of the environment with every head movement. Complete adaptation means, of course, that this motion is no longer seen, but this happened only to one of our $12 \mathrm{Ss}$ within the $6 \mathrm{~h}$ adaptation period. The others still reported apparent field motions at the end of the adaptation period. That they had partially adapted could be shown after removal of the spectacles: facing again the normal displacement of the visual field relative to the turning head, they saw. the field move against the head rotation. The degree to which they had adapted, however, could be ascertained only by a measurement of their no-motion ranges, which were now to be found somewhere among the displacement ratios with the head movement. Such a no-motion range could be compared with an earlier measurement taken before the adaptation period. The adaptation effect was computed for each $\mathrm{S}$ as the difference between the midpoints of these two no-motion ranges. The average adaptation effect for our 12 Ss amounted to $17.5 \%$ DR. For none of them did the two no-motion ranges overlap.

The following explanation will lead to a fuller understanding of this result. Since complete adaptation means that the field displacement of $34 \%$ DR with the head rotation caused by the spectacles produces perception of a stationary environment, after removal of the spectacles an objective target displacement of $34 \%$ DR with the head rotation produced on our measuring apparatus should also lead to perception of a motionless target. This was the case for the one $S$ who had achieved full adaptation. Partial adaptation means that a target moving with the head rotation at some displacement ratio of less than $34 \%$ DR should appear to be motionless; the value of this displacement ratio can likewise be determined with our apparatus. (That our measurements typically yield a range of displacement ratios that cause apparent target rest does not change matters in principle.)

Being able to measure partial adaptation accurately has the advantage that the adaptation period can be shortened considerably and thereby make detailed experimental investigation of the adaptation process possible. We had six Ss wear .66 power spectacles for $2 \mathrm{~h}$ and obtained a mean adaptation of $12.5 \% \mathrm{DR}$ (Wallach \& Kravitz, 1965b). A more radical shortening of the 
adaptation period was achieved by having $S$ turn his head back and forth incessantly. We believed that exposure to displacement of the visual field during head movements is the immediate cause of the developing adaptation and assumed that an incessant exposure to this condition would lead to rapid adaptation. A corresponding strategy had proved successful in adaptation experiments on stereoscopic vision (Wallach \& Karsh, 1963). It succeeded again when $S$, his head attached to the test apparatus, observed the target spot being objectively displaced with each head movement. With a strong target displacement against the head movement of $150 \% \mathrm{DR}$, an adaptation period of $10 \mathrm{~min}$ produced a mean adaptation effect of $13.7 \%$ DR.4

There are two possible reasons for the very rapid adaptation that occurred on these experiments: the high displacement ratio of $150 \%$ which was used here in the training period and the continuous head movements representing a concentration in time of the specific condition of adaptation. As yet unpublished work by Wallach and Frey rules out the high displacement ratio as reason for the very rapid adaptation. Rapid training was given under various conditions, with the displacement ratio one of the variables. No increased adaptation effect was obtained when the displacement ratio was raised from $40 \%$ to $80 \%$. Continuous head turning seems to be the decisive factor. The brief and intensive training condition, however, has its disadvantages also: the adaptation effect does not last as long as one produced in a longer adaptation period, thus making an abbreviated measuring procedure necessary. In the present experiments the training period combined the advantages of continuous head turning and of a longer exposure.

\section{THE PROBLEM}

The experiments here to be reported represent a first step in an analysis of the nature of our adaptation process. We have seen that two kinds of sensory processes are involved in any measurement of the no-motion tange: visual processes representing the displacement of the visual field relative to the head and kinesthetic processes representing extent and probably also rate of the head movement. The process modification which is the result of the adaptation may have, then, any one of three locations; the modification may be in the visual or the kinesthetic process, or there may be a modification of the interaction process where information about the head rotation is taken into account in the perception of motion or rest of the environment. The present experiments ask whether the adaptation we achieve consists in a modification of the processes concerned with kinesthetic stimulation. To answer this question we learned to measure the auditory analogue to the constancy of visual direction (CVD), namely, a constancy of auditory direction, and tested whether adaptation in CVD would transfer to the constancy of auditory direction. If our adaptation is a modification of the kinesthetic processes, that is, if there is a change in the evaluation of the kinesthetic stimulation representing the head rotation, such a transfer should take place.

\section{EQUIPMENT}

The apparatus with which the constancy of visual direction was measured consisted mainly of a variable transmission of the disk and ball type which was mounted above S's seat. The input shaft was vertical, pointing downward, and the horizontal output shaft forward. Attached to the control shaft, with which the position of the ball on the disk is set and hence the transmission ratio is determined, were a disk for turning the shaft and a rotation counter from which the ball position and, after calibration, the transmission ratio could be read. The output shaft terminated in a right angle gear whose vertical end shaft provided the mounting for a front surface mirror. In our apparatus a vertical shaft to which S's head could be fixed was connected to the input shaft of the transmission by a pair of gears which reduced the rotation rate by a factor of five. This was done to diminish the torque needed to operate the transmission. We have since determined that higher torques (of $8 \mathrm{in}$. oz) can be sustained and the head shaft may be attached directly to the transmission; down-gearing could then be provided on the output side of the transmission. This is preferable because the transmission is used more effectively at the higher input rate. Down-gearing by a factor of 10 is recommended. $\mathrm{S}$ wore a stripped down welder's headgear with a vertical plate fixed to the top in median orientation. This plate could be inserted into the slotted end of the input shaft, permitting the head to be shifted in the front-back dimension relative to the shaft until the latter coincided with the axis of S's head rotation before the plate was fastened in the slot.

A homogeneous white screen was erected $2 \mathrm{~m}$ in front of $\mathrm{S}$ who was seated beneath the transmission. A small lantern aimed at the mirror that was connected to the output shaft projected via the mirror a luminous disk $7 \mathrm{~cm}$ in diameter on the screen. When $S$ turned his head back and forth the disk moved back and forth horizontally across the screen, at a rate relative to the head rotation (displacement ratio) that depended on the ratio for which the transmission was set. The lantern and the mirror were so arranged that the target disk was at S's eye level and straight in front of $S$ when his head pointed straight ahead. The transmission could be set to shift the target disk either in the direction with the head rotation or against it. The calibration of the rotation counter, whose reading represents the input/output ratio for which the transmission is set, may be done empirically by measuring the rotation angle of the mirror shaft for one or more full rotations of the input shaft. The ratio of the two rotation angles of the shafts must be doubled to obtain the displacement ratio of the target disk because the reflected lantern beam shifted with twice the rotation angle of the mirror.

Except for the target disk and a small lamp illuminating the counter only, the room was completely dark during the CVD measuring. It is essential that no reference points for target displacement be visible, for they would cause veridical perception of target motion on the basis of object-relative displacement. The disk itself must be kept so dim that it does not serve as a source of illumination for objects in S's visual field.

In our first experiment (Wallach \& Kravitz, 1965a) adaptation training was with 66 power minifying lenses which were essentially reversed Galilean telescopes and were built in our shop. For a reason to be mentioned below, the present experiment employed adaptation to magnification. Commercial lenses of 1.8 power were attached to a welder's headgear with mountings that permitted adjustment to $S$ 's interocular distance and made it possible to bring the lenses close to S's eyes. During the training period $S$ watched television broadcasts while turning his head back and forth more or less continuously. The TV set was almost $4 \mathrm{~m}$ from $S$. The number of head movements $S$ made during the training period was counted in the following fashion. Two photocells, each fitted into the end of a $4 \mathrm{~cm}$ long tube to make their response more directional, were attached to the rear of the headgear. The tubes were positioned to form an angle of $25 \mathrm{deg}$ with each other, with the axis of the head rotation as the apex. A long box containing a fluorescent tube lamp behind a narrow slit which was parallel to the tube was placed in vertical orientation behind $\mathrm{S}$. When he turned his head back and forth, light from the slit alternately activated the two cells. An arrangement of relays tripped an electric counter each time both cells had become activated in succession. A training period lasted $1 \mathrm{~h}$.

The device for measuring the constancy of auditory direction was quite similar to the apparatus used to measure CVD. An identical transmission, again equipped with a rotation counter on the control shaft, was used. Instead of turning a mirror which then caused the visual target to become displaced the output shaft of the transmission turned a rotary switch which would shift an auditory signal through a row of 30 small speakers, 1-1/4 in. diameter. To insure smooth shifting of the sound from one speaker to the next, the moving contact on the rotary switch was wide enough to connect with two speaker contacts during passage from one contact to the next. The speakers were mounted in a horizontal board $3 \mathrm{~m}$ in front of $\mathrm{S}$, in a staggered arrangement so that their angular distances as measured from $S$ amounted to $1 / 2$ deg. S's headgear was attached directly to the input shaft of the transmission. Thus, when $S$ turned his head the signal was 
switcled from speaker to speaker and $E$ could hear it shift along the row of speakers. The rate of that shift relative to the head rotation was controlled by the setting of the variable transmission, and, after calibration, the ratio of the angular displacement of the signal to the angle of the head rotation could be ascertained by reading the counter. The signal consisted of a series of condenser-discharge clicks occurring at a rate of about 10 per sec.

The measurements of CAD took place in a soundproof room with floor and walls heavily padded so that reverberations were greatly diminished. Since our plan was to test for transfer of adaptation acquired by visual training to sound localization, $C A D$ measurements had to be made in the dark. Preliminary measurements had shown that CAD deteriorates rapidly when $S$ is kept in the dark for longer periods; therefore our measuring was frequently interrupted for lighting up the room. Ten sec periods during which the click series was presented alternated with $6 \mathrm{sec}$ periods during which the room lights were turned on. The switching was done by a pair of tandem timers located outside the experimental room.

\section{MEASURING THE CONSTANCY OF VISUAL DIRECTION}

As in our previously reported experiments, the constancy of visual direction and its adaptation were measured by determining the location and the extent of the uncertainty range before and after the adaptation period. The uncertainty range comprised those displacement ratios for which the responses of an $S$ were inconsistent such that, for a given setting of the transmission, different responses were given on repeated trials. The computed midpoint of the uncertainty range was the no-motion point, and the difference between the no-motion points obtained before and after the adaptation period measured the adaptation effect.

The procedure for measuring the uncertainty range was as follows: Displacement ratios were varied in $1 \%$ steps, with each step representing a trial. On each trial, $S$ had to turn his head back and forth three times and reported afterwards whether he had seen the target move or not, and, if he did, in which direction, with the head rotation or against it. The steps were presented in the sequence of the DR scale in two different orders. One order started with a displacement ratio that consistently produced reports of target motion in directions with the head rotation and the other with DRs producing the opposite reports. The initial step was followed by a trial sequence of diminishing DRs until the objective no-motion point was reached where the target spot remained physically stationary. Beyond that point DRs increased again, but now into the range of relative target displacements of opposite direction. To measure an individual uncertainty range $S$ was presented once with both orders. Our procedure deviated from the method of limits at two points. On each run, after trials had produced a sequence of no-motion reports, the first trial that yielded a motion report with a reported direction opposite to the one with which the run had begun was repeated. If the repetition produced a no-motion report the next step was also presented twice, and so on until two motion reports in the right direction had been obtained for the same step. The same procedure was observed on the opposite run. The uncertainty range was computed by plotting the results of the two runs together, counting the steps for which inconsistent reports had been obtained, and adding one-half step for each end of the range.

After an adaptation had been achieved, no-motion reports no longer clustered about the objective no-motion point. In the case of adaptation to magnification, where field displacement is against the direction of the head rotation, no-motion reports after the adaptation period were for target displacements against the head rotation. Therefore, the two orders of trials employed to measure the uncertainty range consisted of target displacements of diminishing or of increasing DRs, both against the head rotation. The diminishing DR sequence was presented first. This was done because, on the post-adaptation measurement, the presentation of a displacement ratio leading to an apparent target displacement with the head would be an occasion for unlearning of the established adaptation. By starting the first sequence with displacement ratios resulting in apparent target displacement against the head, the range of no-motion reports could be mapped out with the presentation of displacement ratios leading to perceived target motion with the heid rotation kept at a minimum.

\section{MEASURING THE CONSTANCY OF AUDITORY DIRECTION}

It was possible to measure the constancy of auditory direction in a manner analogous to the measurement of CVD, that is, by determining an uncertainty range. This was, however, not sure at the outset, for a signal being displaced with the head rotation need not be perceived as a moving sound; a stationary source in an elevated direction may be perceived instead. This follows from the work by Wallach (1940) which shows that the change in the angle which the sound direction forms with the axis of the ears occurring during a head movement is a condition of stimulation in sound localization carrying information about the elevation of the source above the horizontal plane. With the source of sound remaining in the horizontal plane and for head rotation about a vertical axis, only displacement of the sound direction with the head, however, can produce perception of a stationary sound source. It is for this reason that the present experiment dealt with adaptation to displacement against the head rotation. If that adaptation were to transfer to sound localization, an auditory signal normally perceived in motion against the head would have been heard as stationary. The reason why in our experiment displacement of the auditory signal with the head rotation also resulted in apparent signal motion in the horizontal is probably that displacement ratios were small relative to those that had been found effective in sound localization and that the intermittently visible row of loud-speakers suggested localization in the horizontal plane.

Our procedure of measuring the uncertainty range in the constancy of auditory direction was identical with the CVD measurement described, except that the steps on which displacement ratios were varied were more widely spaced, namely, $2.2 \%$ DR instead of $1 \%$ DR. This was done because, understandably, the auditory uncertainty range was larger than the visual one. Care was taken that Ss never turned their heads while the room lights were on, because seeing a stationary environment when the head turns serves to diminish the visually produced adaptation effect. For a similar reason, E never spoke to $S$ while $S$ was turning his head. At the end of the post-adaptation auditory test most Ss were asked to turn their heads while the room was lighted. All of them reported apparent motion of the environment in the direction with the head rotation, an indication that the visual adaptation effect was still operative at the end of the auditory test.

\section{THE PLAN OF THE EXPERIMENT}

The constancy of auditory direction was measured for 24 Ss and the individual uncertainty ranges were computed. Those Ss with the largest uncertainty ranges were excluded from the transfer experiment. The remaining 14 Ss first underwent adaptation to 1.8 power magnification and were tested visually. At this point, five more Ss who had achieved only small adaptation effects were eliminated. The remaining nine Ss later on repeated the adaptation training and were given the auditory test. In our previous work the effect of adaptation was measured as the difference between the no-motion points determined before and after the adaptation period. In the present experiment, the pre-adaptation measurement was taken at the end of a $1-h$ period of TV watching and of continuous head turning identical with the real adaptation training except that the magnifying spectacles were not over S's eyes. Even the head gear was worn and the head movements were counted during these control sessions. The Ss who completed the experiment attended a total of four experimental sessions which were spaced at least three days apart. The first was a control session that ended with the visual test. Then followed a training session culminating in the visual test. Next came again a control session leading to the auditory test. Finally there was a training session that ended with the auditory test. 
During these sessions, which always lasted $1 \mathrm{~h}, \mathrm{~S}$ was under instruction to watch the TV screen and to turn his head back and forth frequently. E checked the head movement counter from time to time and made sure that $S$ moved his head of ten enough to complete a minimum of 2000 pairs of a left and a right turn during the session. Because our experiment involved an auditory test, the sound of the TV broadcast was not turned on. A conflict between the magnification-caused visual target displacement and the immobility of the auditory signal was thus avoided.

\section{RESULTS AND DISCUSSION}

One $h$ of more or less continuous head turning did not have a detrimental effect on the visual uncertainty range. The measurement taken after the first control session resulted in a mean of $3.86 \%$ DR (14 Ss), quite small in comparison with the previously obtained mean of $6.6 \%$ DR (Wallach \& Kravitz, 1965a). The no-motion points, i.e., the midpoints of the uncertainty ranges, seemed also unaffected; their mean amounted to $.58 \% \mathrm{DR}$ in the direction with the head rotation. In our earlier work with rapid adaptation, where training had consisted in $5 \mathrm{~min}$ of continuous head turning, a control experiment had resulted in a mean no-motion point of $4.8 \%$ DR with the head rotation (Wallach \& Kravitz, 1965b). In that experiment, however, the control condition had prevented adaptation by having Ss keep their eyes closed while turning their head continuously, whereas in the control session under discussion Ss viewed television without the magnifiers that normally caused the adaptation. It appears that continuous head moving as such has no appreciable effect on CVD unless eyes are kept closed.

Next to be discussed are the results of the adaptation training measured visually. The mean uncertainty range measured after the first training session was $5.07 \% \mathrm{DR}$, larger by $31 \%$ than after the first control session, but not significantly so. The mean no-motion point after the training period was $9.39 \%$ DR against the head movement, and the adaptation effect measured as the mean difference between the no-motion points obtained after the training session and after the control session was $9.97 \%$ DR. For the nine Ss selected to complete the experiment, the uncertainty range after the first training session was $5.62 \% \mathrm{DR}$, the mean no-motion point was $12.65 \% \mathrm{DR}$, and the mean adaptation effect amounted to $13.22 \%$ DR.

For the constancy of auditory direction the uncertainty range was, expectedly, much larger than the one for CVD. Its mean for the initial measurement taken with the 24 unselected Ss amounted to $29.2 \%$ DR. If only the nine selected Ss who completed the experiment are considered, the initial mean uncertainty range was $22.4 \%$ DR. The measurement taken after the second control session showed a still smaller mean uncertainty range of $17.27 \%$ DR, most likely the practice effect of being tested a second time. There is a good reason for the auditory uncertainty range to be from four to eight times larger than the visual one. Auditory localization is very much less accurate than visual localization. Pierce $^{3}$ found that the least perceptible extent of motion of a well localized auditory signal starting from the median position was 2.5 deg, while Shaffer and Wallach (1966) obtained visual extent-of-motion thresholds under comparable conditions (for a single luminous target in total darkness) that ranged from 1.4 to $4.4 \mathrm{~min}$, a sensitivity about 50 times greater.

The auditory no-motion point did not coincide with objective immobility of the signal. In the initial measurement on 24 Ss it amounted to $3.40 \%$ DR in the direction with the head movement, significantly different from zero at the .05 level. A similar value of $3.15 \%$ DR was obtained after the second control session. We believe that this result is due to the ambiguity, in our experiment, of signal displacements in the direction with the head rotation, which we mentioned above. Such displacements may lead either to apparent target motion or to perception of a stationary sound source elevated above the horizontal plane. The existence of the latter option should have the effect of widening the mean range of no-motion judgments in the direction of displacement with the head movement. In vision, a similar failure of the no-motion point to coincide with objective target rest has a different reason. There, an objectively stationary target at a finite distance from $\mathrm{O}$ is being displaced relative to his eyes in the direction against the head rotation (beyond the normal displacement of a stationary object relative to the head) because the eyes, being located forward from the head's rotation axis, become displaced with the head rotation. This additional displacement of a stationary target due to the location of the eyes is the greater the nearer the target (Wallach \& Kravitz, 1965a). It would cause the no-motion point to be among the target displacement ratios with the head rotation when the uncertainty range is measured. It does not seem likely that our pre-adaptation measurements would show the full effect of this additional displacement. The least that can be expected here is that Ss come to our experiment adapted to some finite field distance and that it is the difference between the latter and the target distance in the apparatus in which the uncertainty range is measured that causes the no-motion point not to coincide with objective target rest. Since the ears are not being laterally displaced by a head turning, this explanation does not apply to the auditory case.

The main question raised in this article, whether an adaptation in CVD transfers to the constancy of auditory direction, was to be answered by the auditory test that followed the second training session. Here, the mean no-motion point was $.75 \% \mathrm{DR}$ in the direction against the head rotation, while at the end of the control session the no-motion point had been $3.15 \%$ DR with the head rotation. The mean of the difference scores between these measurements amounted to $3.85 \%$ DR against the head rotation; it was, however, not significantly different from zero $(p=.2)$. This mean is also quite small compared to the adaptation effect measured with the visual test. The latter amounted to $13.22 \%$ DR and was significantly different from the auditory difference of $3.85 \%$ DR at the .02 level.

We find, then, that partial adaptation in CVD to the effect of magnification was not reflected to an important extent in the measurement of the constancy of auditory direction. Little, if any, of the adaptation in CVD could be attributed to a modification of kinesthetic processes representing head rotation.

\section{REFERENCES}

GIBSON, J. J. Adaptation, after-effect and contrast in the perception of curved lines. Journal of Experimental Psychology, 1933, 16, 1-31.

SHAFFER, O., \& WALLACH, H. Extent-of-motion thresholds under subject-relative and object-relative conditions. Perception \& Psychophysics, $1966,1,447451$.

WALLACH, $H$. The role of head movements and vestibular and visual cues in sound localization. Journal of Experimental Psychology, 1940, 27, 339-368.

WALLACH, H., \& KARSH, E. B. Why the modification of stereoscopic depth perception is so rapid. American Journal of Psychology, 1963, 76, 413-420.

WALLACH, H., \& KRAVITZ, J. H. The measurement of the constancy of visual direction and of its adaptation. Psychonomic Science, 1965a, 2, 217.218.

WALLACH, H., \& KRAVTZ, J. H. Rapid adaptation in the constancy of visual direction with active and passive rotation. Psychonomic Science, $1965 \mathrm{~b}, 3,165-166$.

\section{NOTES}

1. The work was supported by Grant 11089 by the National Institute of Mental Health.

2. Address: Department of Psychology and Education, Swarthmore College, Swarthmore, Pa. 19081, and Department of Psychology, Howard University, Washington, D.C. 20001.

3. The same apparatus was used in the present experiment; for a description see below under Equipment.

4. Such rapid adaptation makes it possible to test whether passive rotation also leads to adaptation. $S$ was placed on a turning chair and, instead of turning his head, he was turned back and forth through an appropriate angle by $\mathrm{E}$. This form of training produced a mean adaptation of 9.8\% DR which was measured with $S$ actively turning his head during the test.

5. Quoted in Robert S. Woodworth: Experimental psychology (1938). P. 519.

(Accepted for early publication July 30, 1968.) 